\begin{tabular}{|c|c|}
\hline \multirow[t]{5}{*}{ Citation/Reference } & Zink, R., Hunyadi, B. ; Van Huffel, S. ; De Vos, M. 2015 \\
\hline & Classifying the Auditory P300 using mobile EEG recordings without \\
\hline & calibration phase. \\
\hline & 37th Annual International Conference of the IEEE Engineering in \\
\hline & Medicine and Biology Society of the IEEE (EMBS) \\
\hline Archived version & $\begin{array}{l}\text { Author manuscript: the content is identical to the content of the } \\
\text { published paper, but without the final typesetting by the publisher }\end{array}$ \\
\hline Published version & Not yet available \\
\hline Journal homepage & http://embc.embs.org/2015/ \\
\hline \multirow[t]{2}{*}{ Author contact } & rob.zink@esat.kuleuven.be \\
\hline & +3216327683 \\
\hline IR & Klik hier als u tekst wilt invoeren. \\
\hline
\end{tabular}




\title{
Classifying the Auditory P300 using mobile EEG recordings without calibration phase.*
}

\author{
R. Zink, B. Hunyádi, S. Van Huffel, and M. De Vos
}

\begin{abstract}
One of the major drawbacks in mobile EEG Brain Computer Interfaces (BCI) is the need for subject specific training data to train a classifier. By removing the need for supervised classification and calibration phase, new users could start immediate interaction with a BCI. We propose a solution to exploit the structural difference by means of canonical polyadic decomposition (CPD) for three-class auditory oddball data without the need for subject-specific information. We achieve this by adding average event-relatedpotential (ERP) templates to the CPD model. This constitutes a novel similarity measure between single-trial pairs and knowntemplates, which results in a fast and interpretable classifier. These results have similar accuracy to those of the supervised and cross-validated stepwise LDA approach but without the need for having subject-dependent data. Therefore the described CPD method has a significant practical advantage over the traditional and widely used approach.
\end{abstract}

\section{INTRODUCTION}

Brain-Computer-Interfaces (BCI) allow users to control a device by thought alone. Although several measures are able to constitute such an interface (e.g. fMRI, NIRS), EEG is the most popular modality to date, mostly due to its relative low cost and high temporal resolution. Moreover the emergence of mobile EEG systems removed the restriction of BCIs to be used in a laboratory setting. Nowadays the field of mobile EEG is emerging rapidly as is evident from the large number of new applications on the one hand, and hardware developments on the other $[1,2,3]$.

The acceptance of these BCIs in mobile settings depends largely upon their accuracy and ease of use. Many machinelearning methods for BCI extract meaningful features and optimize a classifier function based on training data of the test-subject. The information transfer rate, given in bits per trial, is used as an evaluation measurement to rank a method's time-efficiency [2]. Although this is meaningful to compare supervised classifiers, these measures do not always take into account the magnitude of training data needed for the classifier to be trained. Recently there has been increased interest for calibration free BCI methods $[4,5,6]$. These could be especially beneficial for short-term interactions.

* Research supported by Research Council KUL: GOA/10/09 MaNet, CoE PFV/10/002 (OPTEC); PhD/Postdoc grants; Flemish Government, FWO projects: G.0427.10N; EU: ERC Advanced Grant: BIOTENSORS (nr. 339804). This paper reflects only the authors' views, and the Union is not liable for any use that may be made of the contained information.

R. Z., B. H and S. V. H. are with KU Leuven, Department of Electrical Engineering (ESAT), STADIUS Center for Dynamical Systems, Signal Processing and Data Analytics, Kasteelpark Arenberg 10, 3001 Heverlee, Belgium and iMinds Medical IT, (e-mail: rob.zink@esat.kuleuven.be, bori.hunyadi@esat.kuleuven.be and Sabine.VanHuffel@esat.kuleuven.be).

M. D. V. is with the Engineering Department, Oxford University, Oxford United Kingdom (e-mail: maarten.devos@eng.ox.ac.uk) and Cluster of Excellence Hearing4all, University of Oldenburg, Germany.
In this paper we propose a new data driven approach to classify the P300 component as apparent in a three-class auditory oddball paradigm. Focusing attention to either a low or high tone generates (in most cases) a P300 ERP most dominant at central posterior electrodes [7]. We aim to remove the need for subject specific training data in the classification process by incorporating a subject independent stimulus response template in a Canonical Polyadic Decomposition (CPD). The latter has been shown to be able to derive meaningful estimates for large datasets without label information [8,9]. We extend this approach to the classification of single trial pairs of target and non-target (i.e. high-low tone stimuli).

We hypothesize that the incorporation of subjectindependent a priori knowledge about the stimulus protocol and expected responses into data-driven CPD models will allow classification of single trial ERPs. We propose a CPD model with inclusion of average ERP templates to enhance the separability of target and non-target trials with respect to the template. We validate our methods on three-class auditory oddball data to classify 94 target-non-target trial pairs per subject, for twenty subjects. CPD models require a specific rank and initialization that is difficult to determine under these conditions [8]. Therefore, in addition, this approach aims to reduce the need for specific parameter optimization. Our results are compared to those of step-wise linear discriminant analysis (swLDA), a prominent classifier for the P300 ERP. Lastly, we depict possible improvements of the current model taking into account the apparent limitations for generalization to other BCI paradigms.

\section{DATA AND METHODS}

\section{A. Data Acquisition}

Twenty healthy subjects (mean age 24.6 years) performed a three-class oddball auditory task. All participants signed informed consent prior to participation and received monetary reimbursement. The ethics committee of the University of Oldenburg approved the study. This data is obtained from [2].Two deviant $62 \mathrm{~ms}$-tones of $600 \mathrm{~Hz}$ and $1200 \mathrm{~Hz}$, and one standard $900 \mathrm{~Hz}$ tone were played back randomly (ISI $1000 \mathrm{~ms}$, Jitter 0-375 ms). The task of the participants was to silently count these target tones (ten subjects were instructed to pay attention to the $1200 \mathrm{~Hz}$ tone and the other ten to the $600 \mathrm{~Hz}$ tone), ignoring the other two tones. Ninety-four target deviants, 504 standards and 94 deviants that were non-targets were presented randomly while each participant was seated outdoors on a chair. Control of the experiment and delivery of the stimulus was achieved through utilization of OpenViBE software running on a laptop computer. An Emotiv (www.emotiv.com) EEG system with modifications was used to perform data acquisition as described in $[3,10]$. Sintered $\mathrm{Ag} / \mathrm{AgCl}$ 
electrodes were placed at the 10-20 positions $\mathrm{FPz}, \mathrm{F} 3, \mathrm{Fz}$, F4, C3, Cz, C4, TP9, TP10, P3, Pz, P4, O1, and O2.

\section{B. Pre-Processing}

MATLAB and EEGLAB [11] were utilized to analyze the EEG data offline. A semi-automatic attenuation of eye-blink artifacts was achieved through extended infomax ICA (Independent Component Analysis) [12]. A $20 \mathrm{~Hz}$ low-pass filter was applied, and an average of TP10 and TP9 was used for re-referencing. Subsequently, TP10 and TP9 were removed. A pre-stimulus baseline correction was applied after epoch extraction (-200 to $800 \mathrm{~ms}$ ).

Additionally the data were down-sampled from $128 \mathrm{~Hz}$ to $30 \mathrm{~Hz}$ before fitting the CPD. This speeds up the CPD and is expected to still capture most of the P300 waveforms [8]. A window of $167-633 \mathrm{~ms}$ after stimulus onset (SO) is set in order to obtain a general discriminative window for the P300. Figure 1 displays the average (of n-1 subjects) Z-scored ERPs of the Target, non-Target and Baseline stimuli at Cz. For each subject we can create such model ERPs based on the average of the other 19 subjects. Similar patterns as those in Figure 1 are observable across subjects indicating that we have set an appropriate window for P300 discrimination.

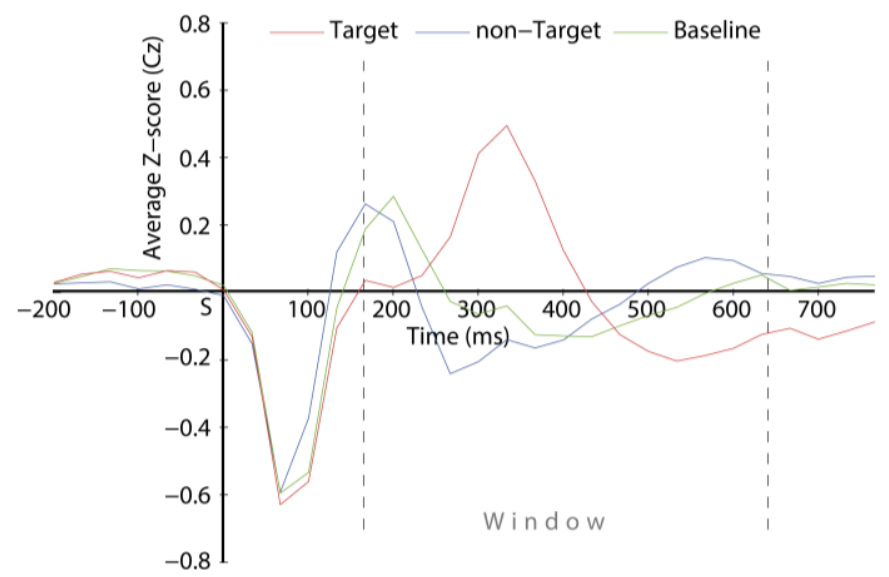

Figure 1: Grand-average (n-1) ERPs at electrode Cz to the Target, nontarget and baseline tone. The vertical lines indicate the general window set for P300 discrimination.

\section{Baseline Template}

The aim of the proposed method is to identify target and non-target trials based on the similarity to a template. This similarity measure is achieved through a data-driven CPD; we reach a rank-1 approximation of the tensor. Including a template ensures that the CPD model will converge more likely to a discriminative space. Evidently, the three stimulus types (i.e. Baseline, Target, non-Target) induce (slightly) different ERP waveforms. Regarding the Targets, no differences were observed between the 600 and $1200 \mathrm{~Hz}$ tone. Moreover, the Baseline and non-Target responses have similar spatio-temporal patterns. As an example, the average (of n-1 subjects) ERP templates for the Baseline, non-Target and Target stimuli are illustrated in Figure 2 from top to bottom respectively. Subtracting the latter two templates from the baseline, we can quantify the average absolute difference (in Z-scores) per time/channel point. The average distance per sample is 0.06 between baseline and non-targets, and 0.19 between baseline and targets. These values confirm that the non-Target stimuli induce a pattern that is similar to that of the baseline, whereas that of the targets is not.

We construct an average Baseline-Template based on 19 subjects. This is done for all 20 subjects to obtain, per subject, a baseline template from non-subject-specific information. This template is used to evaluate the unknown trial pairs through a decomposition using CPD.
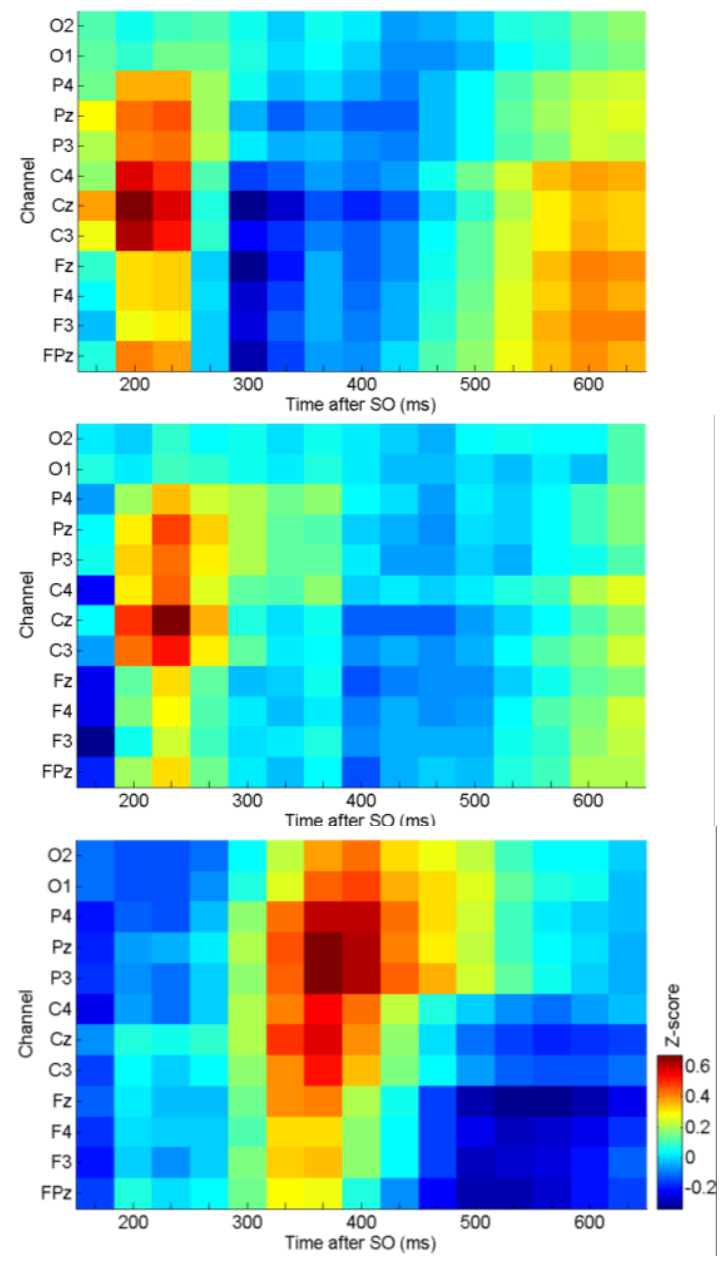

Figure 2: Grand-Average (n-1) heatmap of the spatio-temporal subspace for each of the stimulus types Baseline, non-Targets and Targets, from top to bottom respectively.

\section{D. $C P D$}

Multidimensional signals can be decomposed by the CPD (Canonical Polyadic Decomposition) as a rank-1 tensor sum [13]. For the three-dimensional case the CPD will decompose a tensor $\chi$ as follows:

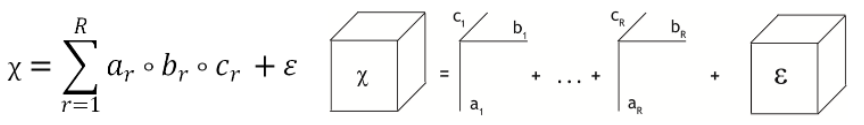

with $R$ representing the number of components, $\mathrm{a}_{\mathrm{r}}, \mathrm{b}_{\mathrm{r}}$, and $c_{r}$ the signatures of every atom in each of the modes, and $\varepsilon$ the model error. Each mode has a specific signature which characterizes every component that is extracted; in the usual three-dimensional tensor of the ERP analysis [channel $\mathrm{x}$ time $\mathrm{x}$ trials], the spatial distribution would be ' $a$ ', the time course would be ' $b$ ', and a given source's trial loadings would be ' $c$ '. The Canonical Polyadic Decomposition model is trilinear, 
which means that each mode's vectors are proportional to each other. In case the data follows a rank-R structure, the decomposition is unique up to permutation and scaling of the extracted components [14].

Since the aim of the oddball paradigm is to induce a P300 to attended stimuli and not to non-targets we aim to construct a data tensor to exploit the brain responses as elicited by the paradigm for a data-driven classifier. For every trial pair we can construct a $12 \times 15 \times 3$ data tensor: 12 channels, 15 time points, and 3 'trials'. The trial dimension consists of the unknown target pair (1 target and 1 non-target) to which we add the Baseline template. The trial loadings (i.e. ' $c$ ' in the equation above) represent the contribution of the time-course on each of these trials. For each tensor we aim for the best rank-1 approximation. The CPD algorithm non-linear least squares is used as implemented in the Tensorlab 2.0 Toolbox [15]. Per subject, for all 94 trial pairs, the baseline template is fixed. Only across subjects do we vary the template according to the averages of the non-subject-data ERPs (as mentioned in section C). Maintaining the structure of the data allows for a decomposition in which we can derive a similarity measure of each trial to the template. This factor vector of the extracted component will provide meaningful information for classification.

\section{E. Classification}

The CPD of the data tensor per single trial will extract 1 component per trial pair. The third factor represents the trial weights accredited to the template and the 2 unknown trials. In order to classify which trial is the Target or non-Target trial we calculate the absolute difference between the trial loading of the template (trial 1) and the two 'unknowns', trial 2 and trial 3. See Figure 5 in the results section for an example. The trial-value with the largest absolute difference from the template is considered the Target trial, the other the non-Target.

swLDA - one of the more commonly utilized algorithms for P300 classification - was compared to the CPD based accuracies for a relevant evaluation. The original set of features comprised seventeen $47 \mathrm{~ms}$ data bins on all twelve electrodes between 0-800 ms. Features are added in sequence when using the swLDA method. The final set of features has a new feature added to it if it improves discrimination of class statistically $\left(\mathrm{p}_{\text {in }}<0.1\right)$. A redundant feature could be removed following the addition of a new feature and a subsequent reappraisal of the current features $\left(\mathrm{p}_{\text {out }}>0.15\right)$ [2]. Although the total number of features utilized is reduced by the stepwise selection of features, a further reduction in overfitting risks was achieved by shrinkage regularization as per BCILAB implementation. The swLDA classification results are obtained with a 5 fold cross-validation procedure. This means that the classifier is trained on $4 / 5$ of the data and tested on the remaining $1 / 5$ which is repeated for different distributions of these parts.

Classification accuracy of single subjects was judged to be over the level of chance significantly if they were found to be higher than a cut-off value. This cut-off was defined as chance-level plus two standard errors, i.e.

$$
0.5+2 \cdot \sqrt{\frac{(0.5 \cdot 0.5)}{188}}=0.573 \text { [16]. Figure } 3 \text { summarizes the }
$$

most important steps in the processing pipeline for both swLDA and CPD.

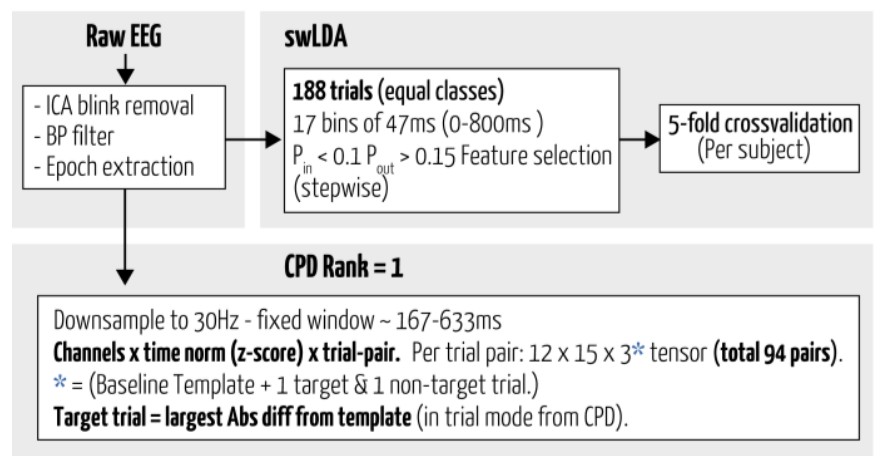

Figure 3. Overview of the most important steps in the analysis for stepwise LDA and CPD.

\section{RESULTS}

\section{A. Classification Accuracy}

Discriminating between the target and non-target trials was above chance level in 19 subjects for swLDA and all subjects for CPD. The average classification accuracy is $70.8 \%(\mathrm{SD}=8.0)$ for swLDA and $70.4 \%(\mathrm{SD}=7.9)$ for $\mathrm{CPD}$. Even though the averages are almost identical, at the single subject level differences can be observed as shown in figure 4 . The correlation between the CPD and LDA results is moderate (Pearson's $\mathrm{r}=.55, \mathrm{p}=.012$, two-tailed). Subjects $1,2,7,17,18,19,20$ have a difference in accuracy larger than $5 \%$ between both methods. Further analysis showed that these differences can largely be explained by the similarity of the target and non-target average data ERPs to the general baseline template - (e.g. subject 17 has an average target ERP that is very different from the baseline template, whereas subject 1 does not).

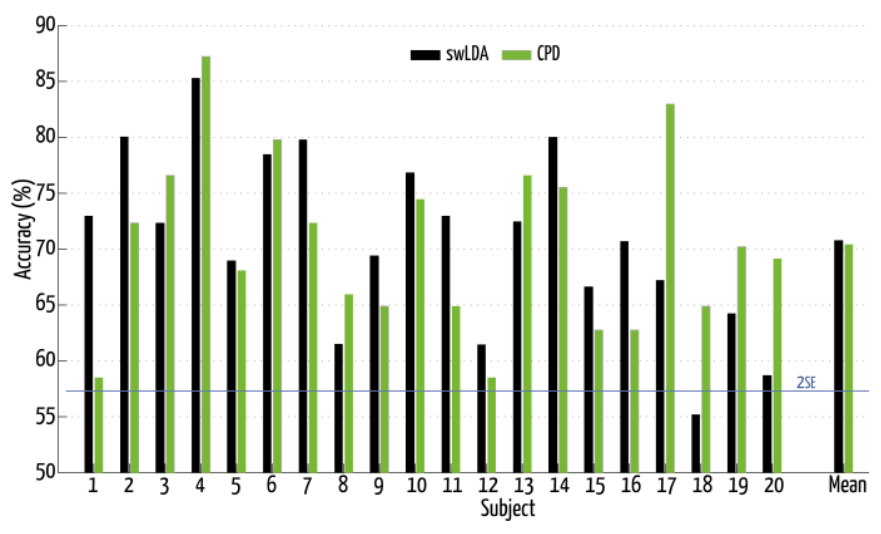

Figure 4: Average classification accuracy for CPD and swLDA for each of the 20 subjects. The blue line represents the above chance-level cut-off (i.e. two standard errors).

\section{B. Single Subject CPD model}

Figure 5 illustrates the results of the average single subject extracted CPD components for subject 3. The first factor represents the distribution over the 12 channels, the second the temporal estimation and the third represents the trial-factor. Note that the (overall) spatio-temporal pattern of the component is able to create a discriminative model for the P300; the temporal mode displays a P300 waveform, and the Target trial deviates the most from the Baseline-Template. 


\section{CPD: Channels $x$ Time Norm x Trials}


Figure 5: Average CPD outcome of subject 3, illustrating the decomposition with the corresponding spatial, temporal and trial mode vectors. $\mathrm{SO}=$ stimulus onset.

\section{CONCLUSION \& DISCUSSION}

Our CPD model does not require subject specific information to obtain classification accuracies comparable to (supervised) swLDA for the classification of auditory oddball BCI data. The largest advantage over swLDA is that we can instantly start with the classification process. The swLDA results are based on using $80 \%$ of the data as training (5-fold cross validation). The average Baseline template used in the CPD models does not take spatial or temporal shifts into account between trials. Nevertheless, our model already seems to achieve an accurate distinction between target and non-target trials.

The moderate correlation between CPD and swLDA results suggests that both methods are classifying the P300 in different ways. The LDA model maximizes the classification accuracy per subject in the feature-weighting step. The CPD model relies strongly on the P300 spatio-temporal structure present at the target-trial (e.g. average in Figure 5). Future study is required to evaluate the relationship of the CPD and LDA classification results more in depth on the single subject and trial level.

Our CPD model performance is based on the P300 waveform differences in an appropriate window set. If for example the N100 ERP was included, the estimated subspace by CPD likely includes the (large) N100 as well, lowering the discrimination power for the P300. Whether the number of channels and or other paradigms lead to similar findings is yet to be discovered.

Two extensions that might improve the current CPD model performance could be to include other templates (e.g. P300 or an artificial signal/noise template), or evaluate a low rank model instead of a rank-1 CPD. The ERP's time and waveform changes between the target and non-target trial could be distinguished more accurately using a Block Term Decomposition (BTD). BTD allows for more flexible estimation of time properties in a so called (L,L,1)decomposition [17] in which the spatial and time dimension are approximated by rank-L components. This might lead to better estimates of signal and noise and thus improve the discriminative subspace of the extracted P300. Similarly, the effectiveness of adaptive models or parameters (e.g. adaptive template, respectively) has yet to be determined.

To conclude, with a simple template based CPD model we are able to competitively classify target/non-target-pairs without the need for a calibration phase. Future studies should focus on extensions of the CPD model, feature/template formations, and the generalization to other paradigms/setups.

\section{REFERENCES}

[1] Mihajlovic, V., Grundlehner, B., Vullers, R., \& Penders, J. (2014). Wearable, Wireless EEG Solutions in Daily Life Applications: What are we missing? IEEE Journal of Biomedical and Health Informatics, vol.PP, no.99, pp.1,1

[2] De Vos, M., Gandras, K., \& Debener, S. (2014). Towards a truly mobile auditory brain-computer interface: Exploring the P300 to take away. International Journal of Psychophysiology, 91(1), 46-53.

[3] Debener, S., Minow, F., Emkes, R., Gandras, K., \& Vos, M. (2012). How about taking a low-cost, small, and wireless EEG for a walk? Psychophysiology, 49(11), 1617-1621.

[4] Kindermans, P. J., Schreuder, M., Schrauwen, B., Müller, K. R., \& Tangermann, M. (2014). True Zero-Training Brain-Computer Interfacing-An Online Study. PloS one, 9(7), e102504.

[5] Grizou, J., Iturrate, I., Montesano, L., \& Oudeyer, P. Y. (2014). Calibration-Free BCI Based Control. In Twenty-Eighth AAAI Conference on Artificial Intelligence (No. EPFL-CONF-198763).

[6] Barachant, A., \& Congedo, M. (2014). A Plug\&Play P300 BCI Using Information Geometry. Technical report arXiv: 1409.0107

[7] Farquhar, J., \& Hill, N. J. (2013). Interactions between pre-processing and classification methods for event-related-potential classification. Neuroinformatics, 11(2), 175-192.

[8] Zink, R., Hunyadi, B., Van Huffel, S., De Vos, M. (2015). Exploring CPD based unsupervised classification for auditory BCI with mobile EEG. Neural Engineering (NER). 7th International IEEE/EMBS

[9] Vanderperren, K., Mijović, B., Novitskiy, N., Vanrumste, B., Stiers, P., Van den Bergh, B. R., .\& De Vos, M. (2013). Single trial ERP reading based on parallel factor analysis. Psychophysiology, 50, 97110.

[10] De Vos, M., Kroesen, M., Emkes, R., \& Debener, S. (2014). P300 speller BCI with a mobile EEG system: comparison to a traditional amplifier. Journal of neural engineering, 11(3), 036008.

[11] Delorme, A., Mullen, T., Kothe, C., Acar, Z. A., Bigdely-Shamlo, N., Vankov, A., \& Makeig, S. (2011). EEGLAB, SIFT, NFT, BCILAB, and ERICA: new tools for advanced EEG processing. Computational intelligence and neuroscience, 2011, 10.

[12] De Vos, M., De Lathauwer, L., \& Van Huffel, S. (2011). Spatially constrained ICA algorithm with an application in EEG processing. Signal Processing, 91(8), 1963-1972.

[13] Harshman, R. A. (1970). Foundations of the parafac procedure: models and conditions for an" explanatory" multimodal factor analysis. Technical report, UCLA Working Papers in Phonetics, 1-84.

[14] Kolda, T. G., \& Bader, B. W. (2009). Tensor decompositions and applications. SIAM review, 51(3), 455-500.

[15] Sorber, L., Van Barel, M., \& De Lathauwer, L. T. (2014).Tensorlab v2. 0. Available online, URL: http://www. tensorlab. net.

[16] Hill, N. J., \& Schölkopf, B. (2012). An online brain-computer interface based on shifting attention to concurrent streams of auditory stimuli. Journal of neural engineering, 9(2), 026011.

[17] Sorber, L., Van Barel, M., \& De Lathauwer, L. (2013). OptimizationBased Algorithms for Tensor Decompositions: Canonical Polyadic Decomposition, Decomposition in Rank- $\left(\mathrm{L}_{\mathrm{r}}, \mathrm{L}_{\mathrm{r}}, 1\right)$ Terms, and a New Generalization. SIAM Journal on Optimization, 23(2), 695-720. 